\title{
Theory of itinerant magnetic excitations in the SDW phase of iron-based superconductors
}

\author{
J. Knolle, ${ }^{1}$ I. Eremin, ${ }^{1,2}$ A.V. Chubukov, ${ }^{3}$ and R. Moessner ${ }^{1}$ \\ ${ }^{1}$ Max-Planck-Institut für Physik komplexer Systeme, D-01187 Dresden, Germany \\ ${ }^{2}$ Institut für Mathematische und Theoretische Physik, \\ TU Braunschweig, D-38106 Braunschweig, Germany \\ ${ }^{3}$ Department of Physics, University of Wisconsin-Madison, Madison, Wisconsin 53706, USA
}

(Dated: September 11, 2018)

\begin{abstract}
We argue that salient experimental features of the magnetic excitations in the SDW phase of ironbased superconductors can be understood within an itinerant model. We identify a minimal model and use a multi-band RPA treatment of the dynamical spin susceptibility. Weakly-damped spinwaves are found near the ordering momentum and it is shown how they dissolve into the particle-hole continuum. We show that ellipticity of the electron bands accounts for the anisotropy of the spin waves along different crystallographic directions and the gap at the momentum conjugated to the ordering one. We argue that our theory agrees well with the neutron scattering data.
\end{abstract}

PACS numbers: 74.70.Xa,75.10.Lp,75.30.Ds

Introduction. The newly discovered iron arsenide superconductors [1] opened a new field in the research on high $T_{c}$ superconductivity. The phase diagram of ferro-pnictide (FP) superconductors is similar to that of layered cuprates and contains an antiferromagnetic phase at small dopings and a superconducting phase at larger dopings. However, parent compounds of iron-based systems are antiferromagnetic metals rather than Mott insulators, in contrast to the cuprates. The electronic structure of parent compounds in the normal state consists of two circular hole pockets of unequal size, centered around the $\Gamma$ point $(0,0)$, and two elliptic electron pockets centered at $(0, \pm \pi)$ and $( \pm \pi, 0)$ points in the unfolded Brillouin zone (UBZ), which is solely based on the $F e$-lattice 2-4]. The dispersions of electron and hole bands are significantly nested, i.e. $\varepsilon_{\mathbf{k}}^{h} \simeq-\varepsilon_{\mathbf{k}+\mathbf{Q}_{\mathbf{i}}}^{e}$ where $\mathbf{Q}_{i}$ is either $\mathbf{Q}_{1}=(\pi, 0)$ or $\mathbf{Q}_{2}=(0, \pi)$. Such nesting is a boost for antiferromagnetism, and several researchers argued that magnetism is itinerant, and at least partly comes from nesting [5 7]. Others argued that magnetism is almost localized and is best described by the $J_{1}-J_{2}$ model [8].

Neutron scattering measurements on parent $F e A s$ compounds revealed that magnetic order consists of ferromagnetic chains along one crystallographic direction in an $F e$ plane and antiferromagnetic chains along the other [9, 10], i.e., the system selects $\mathbf{Q}_{i}$ to be either $\mathbf{Q}_{1}$ or $\mathbf{Q}_{2}$. Sharp propagating spin-wave like excitations have been observed near the ordering momentum (e.g., $\mathbf{Q}_{1}$ ) up to energies of around $100 \mathrm{meV}$, with different velocities along the two crystallographic directions [8, 11]. At larger energies, excitations become overdamped [11]. Such stripe order arises in the $J_{1}-J_{2}$ model of localized spins for $J_{2}<0.5 J_{1}$, and it was argued that such a model can successfully describe some of the experimental data on the spin wave spectra, although, an unusually large in-plane anisotropy of the antiferromagnetic exchange between nearest neighbor spins has to be assumed[8]. However, the same stripe order also appears in the itinerant model as a spin-density-wave (SDW) state with either $\mathbf{Q}_{1}$ or $\mathbf{Q}_{2}$, and is stabilized by the ellipticity of the electron bands and the interactions between the two electron pockets [7]. Furthermore, within the itinerant model, only one hole and one electron Fermi surface (FS) is involved in the SDW mixing. The other two FSs remain intact and give rise to metallic behavior in the SDW phase, even at perfect nesting.

The issue we address here is whether neutron measurements of magnetic excitations can be explained within the itinerant model. This is a crucial test of the itinerant description of magnetism in FPs. A first step in this direction was made in Ref. [6], who analyzed spin excitations in the SDW phase of FPs within a two-band model with circular hole and electron pockets. We argue that to describe anisotropic magnetic excitations in the SDW state one has to consider the model consisting of one circular hole pocket centered around the $\Gamma$-point and two elliptic electron pockets centered around the $(\pi, 0)$ and $(0, \pi)$ points of the unfolded BZ, respectively. Based on this model, we develop a multi-band RPA treatment of the dynamical spin susceptibility and compare the results with available experimental data. We argue that both the high-energy particle-hole continuum and the low-energy propagating excitations can be quantitatively reproduced within our itinerant model. We show that the fact that only one out of two electron pockets is involved in the SDW is responsible for the observed anisotropy of the spin wave excitations along different crystallographic directions.

The model. We depart from a 4-band model, use the experimental fact that the two hole FSs around the $\Gamma$-point have quite different sizes and assume that one hole band interacts with the electron bands much weaker than the other and is thus not important for magnetism. This leads to an effective three band model with one circular hole 


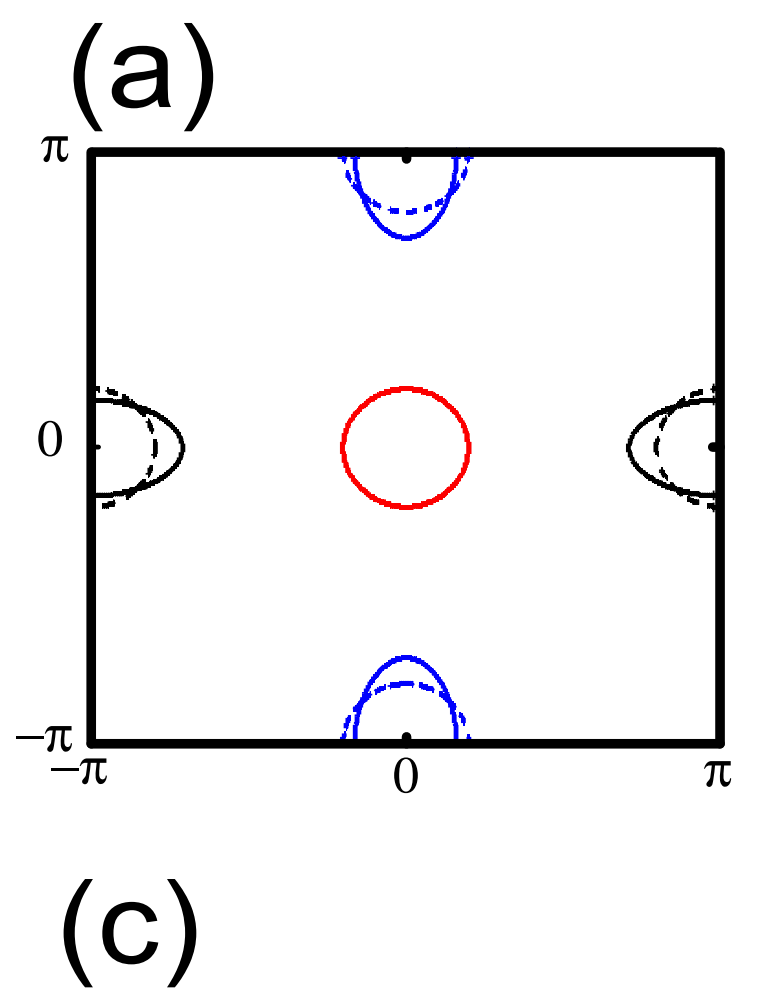

\section{(b)}
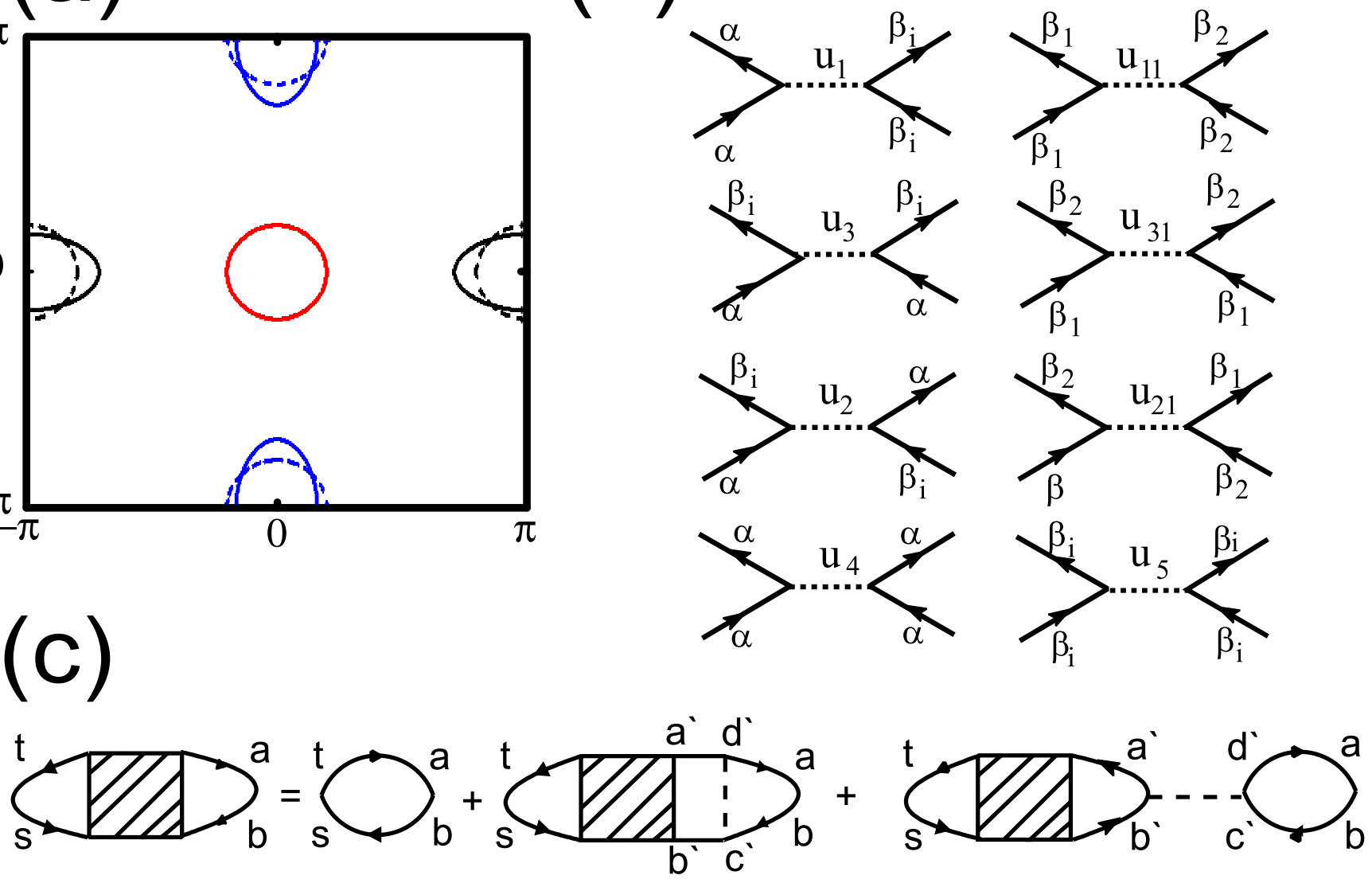

FIG. 1: (color online) (a) Calculated Fermi surfaces for the three band model. The dashed curves refer to the case of $\epsilon=0$, i.e., complete nesting; Diagrammatic representations of the density-density type interactions (b) and the Dyson equation for the RPA spin susceptibility (c) in the three band model.

pocket at $(0,0)\left(\alpha\right.$-band) and two elliptic electron pockets at $\mathbf{Q}_{1}$ and $\mathbf{Q}_{2}(\beta$-bands):

$$
\sum_{\mathbf{p}, \sigma}^{H_{2}=}\left[\varepsilon_{\mathbf{p}}^{\alpha} \alpha_{\mathbf{p} \sigma}^{\dagger} \alpha_{\mathbf{p} \sigma}+\varepsilon_{\mathbf{p}}^{\beta_{1}} \beta_{1 \mathbf{p} \sigma}^{\dagger} \beta_{1 \mathbf{p} \sigma}+\varepsilon_{\mathbf{p}}^{\beta_{2}} \beta_{2 \mathbf{p} \sigma}^{\dagger} \beta_{2 \mathbf{p} \sigma}\right] .
$$

We consider lattice dispersions for all three bands and set $\varepsilon_{\mathbf{p}}^{\alpha}=t_{\alpha}\left(\cos p_{x}+\cos p_{y}\right)-\mu$ and $\varepsilon_{\mathbf{p}}^{\beta_{1}}=\epsilon_{0}+$ $t_{\beta}\left([1+\epsilon] \cos \left(p_{x}+\pi\right)+[1-\epsilon] \cos \left(p_{y}\right)\right)-\mu, \varepsilon_{\mathbf{p}}^{\beta_{2}}=\epsilon_{0}+t_{\beta}\left([1-\epsilon] \cos \left(p_{x}\right)+[1+\epsilon] \cos \left(p_{y}+\pi\right)\right)-\mu$. The parameter $\epsilon$ accounts for the ellipticity of the electron pockets. To make qualitative as well as quantitative comparisons to experiments, we use the Fermi velocities and the size of the Fermi pockets based on Refs. 2, 12]. We obtain $t_{\alpha}=0.85 \mathrm{eV}$, $t_{\beta}=-0.68 \mathrm{eV}, \mu=1.54 \mathrm{eV}, \epsilon_{0}=0.31 \mathrm{eV}$, and $\epsilon=0.5$. For these values, Fermi velocities are $0.5 \mathrm{eVa}$ for the $\alpha$-band, where $a$ is the $F e-F e$ lattice spacing, and $v_{x}=0.27 e V a$ and $v_{y}=0.49 \mathrm{eVa}$ along $x$ - and $y$-directions for the $\beta_{1}$-band (vice versa for $\beta_{2}$, we set $a_{x}=a_{y}=a$ ). The corresponding Fermi surfaces is shown in Fig. 1(a).

The interacting part of the Hamiltonian contains density-density interactions with small momentum transfer and momentum transfers $(\pi, 0),(0, \pi)$, and $(\pi, \pi)$. The interactions contributing to SDW formation are (in the terminology 
of Ref.[13])

$$
\begin{aligned}
& H_{4}=\sum U_{1} \alpha_{\mathbf{p}_{3} \sigma}^{\dagger} \beta_{j \mathbf{p}_{4} \sigma^{\prime}}^{\dagger} \beta_{j \mathbf{p}_{2} \sigma^{\prime}} \alpha_{\mathbf{p}_{1} \sigma}+ \\
& \frac{U_{3}}{2}\left[\beta_{j \mathbf{p}_{3} \sigma}^{\dagger} \beta_{j \mathbf{p}_{4} \sigma^{\prime}}^{\dagger} \alpha_{\mathbf{p}_{2} \sigma^{\prime}} \alpha_{\mathbf{p}_{1} \sigma}+h . c\right] .
\end{aligned}
$$

For simplicity, we treat both $U_{1}$ and $U_{3}$ as constants. Both interactions involve $\beta_{1}$ and $\beta_{2}$ fermions and in general

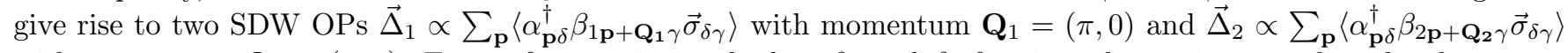
with momentum $\mathbf{Q}_{2}=(0, \pi)$. For perfect nesting and when $\beta_{1}$ and $\beta_{2}$ fermions do not interact directly, the energy depends only on $\vec{\Delta}_{1}^{2}+\vec{\Delta}_{2}^{2}$, i.e., the order is in general a combination of $\vec{\Delta}_{1}$ and $\vec{\Delta}_{2}$ and the ground state is degenerate. However, ellipticity of the electron pockets and a direct interaction between electron bands gives rise to an additional $a_{1} \vec{\Delta}_{1}^{2} \vec{\Delta}_{2}^{2}$ term in the energy, with a positive prefactor $a_{1}$ (Ref. [7]). Then, the energy is minimized when either $\vec{\Delta}_{1}=0$ or $\vec{\Delta}_{2}=0$, i.e., SDW order is ferromagnetic along one direction and antiferromagnetic along the other, in agreement with experiments. Such a state couples $\alpha$ fermions with only one band of $\beta$ fermions, leaving the other band intact, i.e., leaving one of the electron FSs unaffected by SDW.

Without loss of generality we direct $\vec{\Delta}_{1}$ along the $z$-axis and set $\vec{\Delta}_{2}=0$. The resulting mean-field Hamiltonian can be diagonalized by a standard Bogolyubov transformation

$$
\begin{aligned}
\alpha_{\mathbf{p} \sigma} & =u_{\mathbf{p}} c_{\mathbf{p} \sigma}+v_{\mathbf{p}} d_{\mathbf{p} \sigma} \\
\beta_{1 \mathbf{p}+\mathbf{Q}_{\mathbf{1}} \sigma} & =\sigma\left(v_{\mathbf{p}} c_{\mathbf{p} \sigma}-u_{\mathbf{p}} d_{\mathbf{p} \sigma}\right)
\end{aligned}
$$

where $u_{\mathbf{p}}^{2}, v_{\mathbf{p}}^{2}=\frac{1}{2}\left(1 \pm \frac{\varepsilon_{\mathbf{p}}^{\alpha}-\varepsilon_{\mathbf{p}+\mathbf{Q}_{1}}^{\beta_{1}}}{\sqrt{\left(\varepsilon_{\mathbf{p}}^{\alpha}-\varepsilon_{\mathbf{p}+\mathbf{Q}_{\mathbf{1}}}^{\beta_{1}}\right)^{2}+4 \Delta_{1}^{2}}}\right)$. After the transformation, the Hamiltonian $H_{2}+H_{4}$ becomes

$$
H_{S D W}^{d i a g}=\sum_{\mathbf{p}, \sigma}\left[E_{\mathbf{p}}^{c} c_{\mathbf{p} \sigma}^{\dagger} c_{\mathbf{p} \sigma}+E_{\mathbf{p}}^{d} d_{\mathbf{p} \sigma}^{\dagger} d_{\mathbf{p} \sigma}+\varepsilon_{\mathbf{p}}^{\beta_{2}} \beta_{2 \mathbf{p} \sigma}^{\dagger} \beta_{2 \mathbf{p} \sigma}\right]
$$

where

$$
E_{\mathbf{p}}^{c, d}=\frac{1}{2}\left(\varepsilon_{\mathbf{p}}^{\alpha}+\varepsilon_{\mathbf{p}+\mathbf{Q}_{\mathbf{1}}}^{\beta_{1}} \pm \sqrt{\left(\varepsilon_{\mathbf{p}}^{\alpha}-\varepsilon_{\mathbf{p}+\mathbf{Q}_{\mathbf{1}}}^{\beta_{1}}\right)^{2}+4 \Delta_{1}^{2}}\right)
$$

are the new dispersions and the magnitude of the SDW gap is determined self-consistently from $\Delta_{1}=$ $-U_{S D W} \sum_{\mathbf{p}} u_{\mathbf{p}} v_{\mathbf{p}}\left[f\left(E_{\mathbf{p}}^{c}\right)-f\left(E_{\mathbf{p}}^{d}\right)\right]$, where $U_{S D W}=U_{1}+U_{3}$ and $f(E)$ is the Fermi function.

Magnetic Susceptibility and RPA. We will follow earlier works [6, 14] and use a generalized RPA approach. The dynamical susceptibility tensor in the multiband case is defined as

$$
\begin{array}{r}
\chi_{l m}^{s t, a b}\left(\mathbf{q}, \mathbf{q}^{\prime}, i \Omega\right)=\frac{1}{2} \int_{0}^{\beta} d \tau e^{i \Omega \tau} \sum_{\mathbf{p}, \mathbf{p}^{\prime}, \gamma, \delta, \gamma^{\prime}, \delta^{\prime}} \times \\
\left\langle T_{\tau} s_{\mathbf{p} \gamma}^{\dagger}(\tau) t_{\mathbf{p}+\mathbf{q} \delta}(\tau) a_{\mathbf{p}^{\prime} \gamma^{\prime}}^{\dagger}(0) b_{\mathbf{p}^{\prime}-\mathbf{q}^{\prime} \delta^{\prime}}(0)\right\rangle \sigma_{\gamma \delta}^{l} \sigma_{\gamma^{\prime} \delta^{\prime}}^{m}
\end{array},
$$

where $T_{\tau}$ is the time ordering operator and quasiparticle operators $s, t, a, b$ are operators from either $\alpha$ or $\beta_{1,2}$ bands. Because the unit cell is doubled in the SDW state, the susceptibility is nonzero for $\mathbf{q}=\mathbf{q}^{\prime}$ and $\mathbf{q}=\mathbf{q}^{\prime}+\mathbf{Q}_{1}($ Ref. [15]). In terms of Green functions (GF) we have $\chi_{z z}^{s t, a b}(\mathbf{q}, i \Omega)=-\frac{1}{2 \beta} \sum_{\omega_{n}} \sum_{\mathbf{p}, \sigma} G_{\mathbf{p} \sigma}^{b s}\left(i \omega_{n}\right) G_{\mathbf{p}+\mathbf{q} \sigma}^{t a}\left(i \omega_{n}+i \Omega\right)$, and $\chi_{ \pm}^{s t, a b}(q, i \Omega)=-\frac{1}{\beta} \sum_{\omega_{n}} \sum_{\mathbf{p}} G_{\mathbf{p} \uparrow}^{b s}\left(i \omega_{n}\right) G_{\mathbf{p}+\mathbf{q} \downarrow}^{t a}\left(i \omega_{n}+i \Omega\right)$, where $G_{\mathbf{p} \sigma}^{s t}\left(i \omega_{n}\right)=-\int_{0}^{\beta} d \tau\left\langle T_{\tau} s_{\mathbf{p} \sigma}(\tau) t_{\mathbf{p} \sigma}^{\dagger}(0)\right\rangle e^{i w_{n} \tau}$. In order to compute the RPA susceptibility, we also include all other interactions: the exchange interaction between $\alpha$ and $\beta$ fermions and the interactions between electron pockets (see Fig. 1(b)). These interactions do not contribute towards SDW formation but they do play a role in determining the structure of the spin wave excitations.

Spin susceptibilities $\left[\chi_{l m}^{s t, a b}\right]_{R P A}$ in the RPA approximation are obtained via a Dyson equation

$$
\left[\chi_{l m}^{s t, a b}\right]_{R P A}=\chi_{l m}^{s t, a b}+\chi_{l m}^{s t, a^{\prime} b^{\prime}} U_{l m}^{a^{\prime} b^{\prime}, s^{\prime} t^{\prime}} \chi_{l m}^{s^{\prime} t^{\prime}, a b}
$$

and the summation over repeated band indices is assumed. The Dyson equation is schematically shown in Fig:1(c) (depending on $l, m$, the series contain either ladder or bubbles). The solution of Eq.(17) in matrix form is straightforward $\left[\hat{\chi}_{l m}\right]_{R P A}=\hat{\chi}_{l m}\left(1-\hat{U}_{l m} \hat{\chi}_{l m}\right)^{-1}$. For the single band Hubbard model our results agree with those of Ref.[15]. 


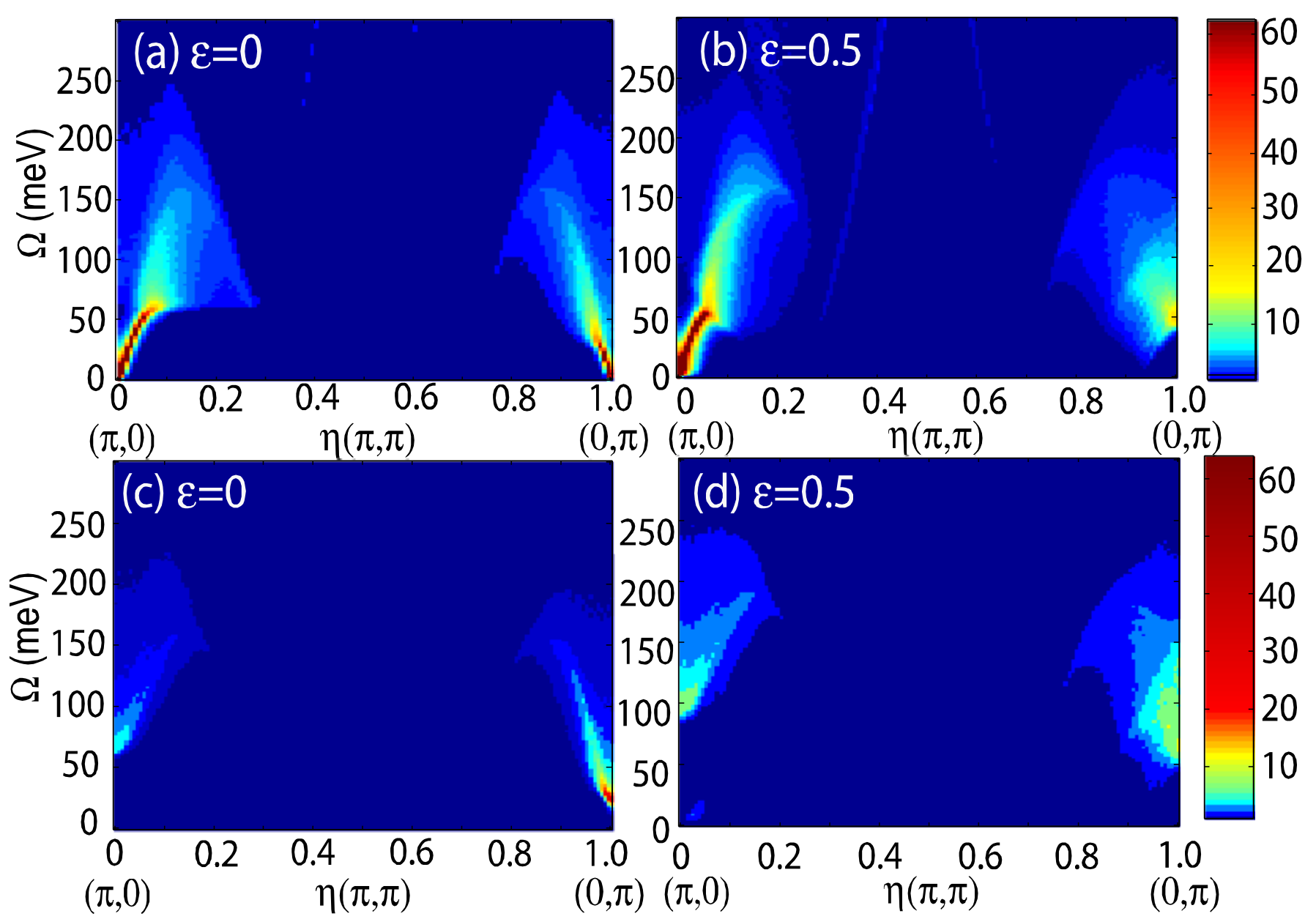

FIG. 2: (color online) Calculated imaginary part of the transverse (upper panel) and longitudinal (lower panel) component of the physical RPA spin susceptibility as a function of frequency and momentum (plotted along $(0, \pi) \rightarrow(\pi, 0)$ points of the BZ) for $\epsilon=0$ (a)-(c), and $\epsilon=0.5$ (b)-(d). The color bars refer to the intensity in units of st./eV. For numerical purposes we set the damping constant $\delta=1 / 300 \mathrm{eV}$.

Results and Comparison to Experiments. We set $U_{S D W} \approx 0.52 \mathrm{eV}$ for zero ellipticity, to match the experimental $T_{N} \sim 200 K$. This yields a $T=0$ value of the SDW gap of $\Delta_{1} \approx 31 \mathrm{meV}$. As the interactions between electron pockets are likely smaller than between electron and hole pockets, we set, for definiteness, $U_{5}=U_{2}=0.5 U_{1}, U_{1}=U_{3}$, and $U_{j 1}=0.1 U_{j}$. We verified that the spin wave dispersion around the ordering wave vector does not change in any substantial way if we vary these numbers. In particular, when the ellipticity parameter $\epsilon \neq 0$, we can set $U_{j 1}=0$ and it will not affect the outcome. The results of our calculations are presented in Fig. 2 for $T=0$ and for several values of the ellipticity parameter $\epsilon$. Consider first the case of circular electron pockets, $\epsilon=0$, which is for magnetic properties the same as full nesting. In this case two out of three FS are completely gapped, if the order parameter exceeds the threshold value, which is the case for our $\Delta$. In Fig 2(a) we show the calculated imaginary part of the transverse component of the susceptibility, $\operatorname{Im}\left[\hat{\chi}_{+-}(\mathbf{q}, \Omega)\right]_{R P A}$ along the direction $(0, \pi)$ to $(\pi, 0)$. Because the corresponding ground state is degenerate, the excitation spectrum has a Goldstone mode at the ordering momentum $\mathbf{Q}_{1}$ and another gapless mode at $\mathbf{Q}_{2}$. This result was earlier found in Ref. [6]. We also clearly see that the excitations near the ordering momentum are propagating up to $\Omega_{c}\left(\mathbf{Q}_{1}\right)=2 \Delta_{1} \sim 60 \mathrm{meV}$ what is expected because both pockets separated by $\mathbf{Q}_{1}$ are gapped. Excitations near $\mathbf{Q}_{2}$ are also propagating, but up to smaller energies, $\Omega_{c}\left(\mathbf{Q}_{2}\right)=\Delta_{1}$, that is consistent with the fact that only one of the two pockets separated by $\mathbf{Q}_{2}$ is gapped. At higher energies spin-waves enter the continuum and become overdamped Stoner-like excitations, however, still with well defined peaks. The difference between $\Omega_{c}\left(\mathbf{Q}_{1}\right)$ and $\Omega_{c}\left(\mathbf{Q}_{2}\right)$ is also seen in Fig.2(c) where we plotted the longitudinal component of the susceptibility. As expected, $\operatorname{Im}\left[\hat{\chi}_{+-}(\mathbf{q}, \Omega)\right]_{R P A}$ vanishes below $\Omega_{c}(\mathbf{Q})$.

At finite $\epsilon=0.5$, we used a slightly larger $U_{S D W}$ to recover the same $T_{N}$ as for $\epsilon=0$, which in turn leads to a slightly larger $\Delta_{1}$. The results are presented in panels (b) and (d) of Fig. 2, There are two key effects introduced by ellipticity. First, the degeneracy is now broken and the transverse excitations near $\mathbf{Q}_{2}$ acquire a finite gap clearly 

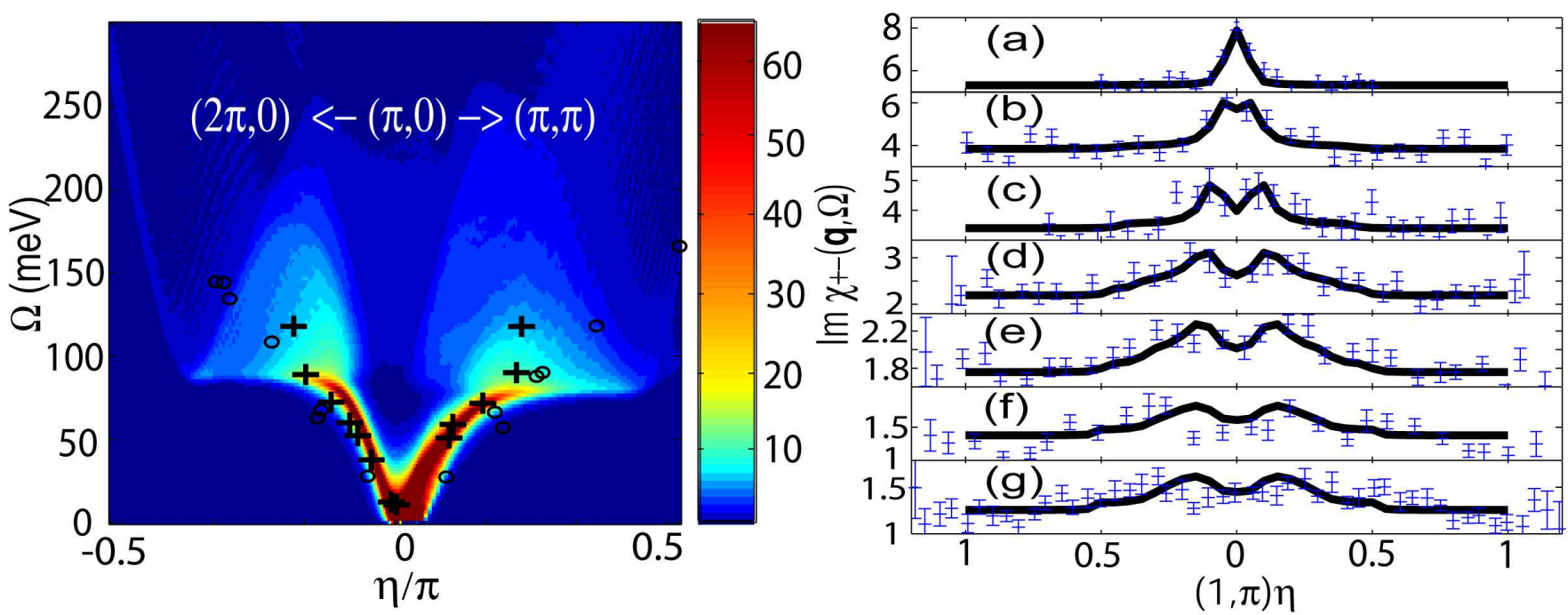

FIG. 3: (color online) Left panel: Calculated $\operatorname{Im} \chi_{R P A}^{+-}$as a function of frequency and momentum (plotted along $(0,2 \pi) \rightarrow$ $(0, \pi) \rightarrow(\pi, \pi)$ directions of the BZ) for $\epsilon=0.5$. The crosses and circles are the measured points taken from Refs. [1] and 8], respectively. Right panel: Calculated constant energy cuts of $\operatorname{Im} \chi^{+-}(\mathbf{q}, \Omega)$ along $(0,0) \rightarrow(\pi, 0) \rightarrow(2 \pi, 0)$ direction $($ solid curves) for 25 (a), 48 (b), 64 (c), 100 (d), 115 (e), 135 (f), and 144 (g) (all in meV). The experimental data are taken from Ref.[8]. The intensity maxima have been adjusted to the experimental values.

visible in Fig2(c). Second, spin-wave excitations near $\mathbf{Q}_{1}$ have a finite Landau damping down to $\Omega=0$ because the SDW order no longer completely gaps the hole and electron FSs separated by $\mathbf{Q}_{1}$. At the same time, spin-wave excitations are still clearly visible at low energies and do not become overdamped. The reasoning is that SDW coherence factors suppress the scattering between $\alpha$ and $\beta_{1}$ fermions and reduce the Landau damping around the ordering momentum $\mathbf{Q}_{1}$ from $\Omega /\left|\mathbf{q}-\mathbf{Q}_{1}\right|$ to $\Omega\left|\mathbf{q}-\mathbf{Q}_{1}\right|$ what for linear dispersion is of the same order as $\Omega^{2}$ and $\left(\mathbf{q}-\mathbf{Q}_{1}\right)^{2}$. Within the one-band model, this effect has been discussed in [16]. Once $\Omega$ exceeds $\Omega_{c}\left(\mathbf{Q}_{2}\right)$, the coherence factors no longer screen Landau damping, and spin-waves become Stoner-like, overdamped excitations. The scattering between $c$ and $\beta_{2}$ fermions is not suppressed by the SDW coherence factors because $\mathbf{Q}_{2}$ is not the ordering momentum, therefore, Landau damping makes the transverse excitations near $\mathbf{Q}_{2}$ overdamped immediately above the gap. Note, that there is also a tendency towards incommensuration near $\mathbf{Q}_{2}$. Longitudinal excitations are still gapped up to $\Omega_{c}(\mathbf{Q})$ and become Stoner-like at higher energies, as is clearly seen in Fig. 2(d).

Despite the fact that our model is indeed a simplification of the actual 5-band model for FPs, it reproduces quite well the experimental data for the magnetic excitations. First, the excitations near the "wrong" momentum $\mathbf{Q}_{2}$ are gapped up to about $50 \mathrm{meV}$ as seen in Fig 2 (c). Second, measured excitations near $\mathbf{Q}_{1}$ are propagating spin-waves up to about $100 \mathrm{meV}$, with a finite but small damping. In the left panel of Fig 3 we compare our dispersion with the experimental data [8, 11]. We emphasize that both the measured and the calculated spectra have different velocities along different crystallographic directions. In our theory, it is a consequence of the non-zero ellipticity and the fact that only one electron pocket is involved in the SDW formation. Remarkably, for $\epsilon=0.5$ the anisotropy is the same as in the experimental data. Another verifiable theory prediction for this range is that the width of the spin-wave peak should scale as $\Omega^{2}$. Third, we find that excitations are still visible even when the spin-waves enter the continuum. In the right panel of Fig 3 we compare the calculated and the measured $\operatorname{Im} \chi^{+-}(q, \Omega)$ along $(0,0) \rightarrow(\pi, 0) \rightarrow(2 \pi, 0)$ direction for different $\Omega$. We see that the agreement is quite good for all frequencies even above $\Omega_{c}$ [17].

Conclusions In this paper we analyzed the structure of the magnetic excitations in the magnetically ordered state of Fe-pnictides. We used a multi-band itinerant model and developed a multi-band RPA treatment of the longitudinal and transverse components of the dynamical spin susceptibility. We found weakly-damped spin-waves near the ordering momentum and showed how they dissolve into the particle-hole continuum above an energy which scales with the SDW order parameter. For perfect nesting between electron and hole bands the SDW state has an extra degeneracy, and we found an extra gapless mode at the momentum different from the ordering one. When ellipticity of the electron bands is included, the degeneracy is lifted and the extra mode becomes gapped. Ellipticity of the electron pockets also naturally accounts for the anisotropy of the spin waves along different crystallographic directions. We argue that our theory agrees well with the available neutron scattering data.

We thank P. Dai and J. Zhao for sending us the experimental data and N. Shannon, M. Vavilov and A. Vorontsov for stimulating discussions. I.E. acknowledges the support from the RMES Program (Contract No. N 2.1.1/3199), 
and NSF (Grant DMR-0456669). A.V.C. acknowledges the support from nsf-dmr 0906953.

[1] Y. Kamihara, T. Watanabe, M. Hirano, and H. Hosono, J. Am. Chem. Soc. 1303296 (2008).

[2] D.J. Singh and M.-H. Du, Phys. Rev. Lett. 100, 237003 (2008); L. Boeri, O.V. Dolgov, and A.A. Golubov, Phys. Rev. Lett. 101, 026403 (2008); I.I. Mazin, D.J. Singh, M.D. Johannes, and M.H. Du, Phys. Rev. Lett. 101, 057003 (2008).

[3] C. Liu et al., Phys. Rev. Lett. 101, 177005 (2008); D.V. Evtushinsky et al., Phys. Rev. B 79, 054517 (2009);

[4] A.I. Coldea et al., Phys. Rev. Lett. 101, 216402 (2008).

[5] V. Cvetkovic and Z. Tesanovic, EPL 85, 37002 (2009); V. Barzykin and L. P. Gorkov, JETP Lett. 88, 131 (2008); F. Wang, H. Zhai, Y. Ran, A. Vishwanath, and D.-H. Lee, Phys. Rev. Lett. 102, 047005 (2009); M. Daghofer, A. Nicholson, A. Moreo, and E. Dagotto, Phys. Rev. B 81, 014511 (2010); P.M.R. Brydon and C. Timm, Phys. Rev. B 79, 180504(R) (2009).

6] P.M.R. Brydon and C. Timm, Phys. Rev. B 80, 174401 (2009).

[7] I. Eremin and A.V. Chubukov, Phys. Rev. B 81, 024511 (2010).

[8] J. Zhao et al., Nature Phys. 5, 555 (2009).

[9] Clarina de la Cruz et al., Nature 453, 899 (2008).

[10] H.-H. Klauss et al., Phys. Rev. Lett. 101, 077005 (2008).

[11] S. O. Diallo et al., Phys. Rev. Lett. 102187206 (2009).

[12] H. Ding et al., arXiv:0812.0534v1 (unpublished).

[13] A. V. Chubukov, D. V. Efremov, and I. Eremin, Phys. Rev. B 78134512 (2008).

[14] S. Graser, T.A. Maier, P.J. Hirschfeld, D.J. Scalapino, New J. Phys. 11, 025016 (2009).

[15] J. R. Schrieffer, X. G. Wen, and S. C. Zhang, Phys. Rev. B 3911663 (1989); A.V. Chubukov and D.M. Frenkel, Phys. Rev. B 46, 11884 (1992).

[16] S. Sachdev, A.V. Chubukov, and A. Sokol, Phys. Rev. B 51, 14874 (1995).

[17] Note that to compare the absolute intensities with experimental data, the structure of the orbital matrix elements is normally required. In general, the full $\chi_{l m}$ is a linear combination of $\left[\chi_{l m}^{s t, a b}\right]_{R P A}:\left[\chi^{l m}(\mathbf{q}, \Omega)\right]=A_{s t, a b}\left[\chi_{l m}^{s t, a b}(\mathbf{q}, \Omega)\right]_{R P A}$ with some matrix elements $A_{s t, a b}$ originating from the transformation of the orbitals to the bands. On the other hand, all $\left[\chi_{l m}^{s t, a b}(\mathbf{q}, \Omega)\right]_{R P A}$ have the same denominator, and hence the structure of the excitations does not depend on the form of $A_{s t, a b}$ (the intensity does, indeed, depend on $A_{s t, a b}$ ). 